\title{
Familiares cuidadores de mayores: autopercepción de los cuidados
}

\author{
Vicente Pérez Cano
}

<vpercan@upo.es>

Rosa Ma Varela Garay

Facultad de Ciencias Sociales, Universidad Pablo de Olavide (Sevilla)

Belén Martínez Ferrer

Facultad de Ciencias Sociales, Universidad Pablo de Olavide (Sevilla)

\section{Gonzalo Musitu Ochoa}

Facultad de Ciencias Sociales, Universidad Pablo de Olavide (Sevilla)

\begin{abstract}
Azterlan honen xedea da mendekotasun-egoeran dauden pertsona edadetuak zaintzen aritzen diren zaintzaile ez formal nagusien egoera pertsonala, familiakoa eta soziala ezagutzea. Lagina osatu dute pertsona edadetu eta mendekoren bat duten Andaluziako zortzi probintzietako 294 familia hautatuz. Analisi faktoriala burutzen da varimax rotazioa duten osagai nagusiak erabiliz. Faktore horien ostean burutzen da aldagai anitzeko bariantza-analisia. Bertan jasotako emaitzek hipotesi orokorra baiesten dute, eta eragin ezkorrak ematen dira maila fisiko, psikologiko, familia eta sozialean mendeko-egoeran dauden pertsonak zaintzen aritzen diren horien artean. Bertan analizatu eta eztabaidatzen dira zientziak egun dakienaren arabera emaitzetan jasotako datuak, eta iradokitzen dira zenbait ideia etorkizunean ikerketa eta ekimenak burutu ahal izateko.
\end{abstract}

\section{HITZ-GAKOAK:}

mendekotasuna, familia, zaintzaile ez formalak, pertsona edadetuak, familiaren zama.
El objetivo del presente estudio es analizar la situación personal, familiar y social de los/as cuidadores/as informales principales, que se dedican $a$ atender a personas mayores con dependencia. La muestra está constituida por 294 familiares que representan a las ocho provincias de la comunidad autónoma de Andalucía, y que se ocupan del cuidado de una persona mayor dependiente. Se realiza un análisis factorial de componentes principales con rotación varimax, A partir de estos factores, se llevan a cabo análisis multivariante de varianza. Los resultados obtenidos confirman la hipótesis general, en el sentido de que el cuidado de las personas en situación de dependencia tiene efectos negativos directos en los ámbitos físico, psicológico, familiar y social de las personas cuidadoras. Se analizan y discuten los resultados obtenidos a la luz del conocimiento científico actual, y se sugieren ideas para investigaciones futuras y para la acción.

\section{Palabras clave:}

dependencia, familia, cuidadores informales, personas mayores, carga familiar. 


\section{Introducción}

La Organización Mundial de la Salud, a través de la Clasificación Internacional de Funcionamiento de la Discapacidad y de la Salud (2001), define la dependencia como "la situación en la que una persona con discapacidad precise de ayuda, técnica o personal, para la realización (o mejora del rendimiento funcional) de una determinada actividad". Las actividades que se consideran son las de comunicación, movilidad y de autocuidado. En este concepto de dependencia, Querejeta González (2003) destaca dos aspectos: la limitación de la actividad y la ayuda externa. La dependencia está condicionada tanto por estados de salud -permanentes o transitorios- como por el medio en el que se desenvuelve la persona en cuestión, de tal manera que, en un contexto determinado una persona puede tener dependencia de alguna ayuda técnica, y en otro contexto, no.

Creemos que la dependencia debe entenderse como "el estado de carácter permanente en que se encuentran las personas que, por razones derivadas de la edad, la enfermedad o la discapacidad y ligadas a la falta 0 a la pérdida de autonomía física, mental, intelectual o sensorial, precisan de la atención de otra u otras personas, así como de ayudas importantes para realizar actividades básicas de la vida diaria, o en el caso de las personas con discapacidad intelectual o enfermedad mental, de otros apoyos para su autonomía personal" (Ley 39/2006, de 14 de diciembre, de Promoción de la Autonomía Personal y Atención a las Personas en Situación de Dependencia, art. 2.2). Para Castón y Ramos (2006), la dependencia es el estado en el que se encuentran personas que, por razones ligadas a la falta de autonomía física, psíquica o intelectual, tienen necesidad de asistencia para realizar las actividades de la vida cotidiana.

Un aspecto esencial que conviene precisar en la dependencia son las actividades de la vida diaria en las que las personas con discapacidad necesitan ayudas. Diversos autores consideran dos tipos de actividades. Las actividades de la vida diaria (AVD) se definen como las tareas más elementales de la persona, que le permiten desenvolverse con un mínimo de autonomía e independencia, tales como el cuidado personal, las actividades domésticas básicas, la movilidad esencial, reconocer personas y objetos, orientarse, y entender y ejecutar órdenes o tareas sencillas. Las actividades instrumentales de la vida diaria (AIVD) son aquellas que contribuyen al mantenimiento de la infraestructura. Por su carácter instrumental, requieren habilidades para mantener el hogar (gestión de las propiedades, preparación de alimentos, tareas domésticas y uso del transporte, entre otras) [Ministerio de Trabajo y Asuntos Sociales, 2006].

En ocasiones, se habla de cuidadores informales, en referencia a una tarea que suele implicar a varias personas (Montoro Rodríguez, 1999), aunque, normalmente, la labor del cuidado se centra en una persona, en la mayoría de los casos el cónyuge, la madre o la hija. Es en esta persona en quien recae la mayor parte de la carga, como demuestran las encuestas y los estudios realizados (Pérez Cano, Musitu Ochoa y Moreno Ruiz, 2011). La actividad del cuidado es muy diversa y depende de las características de la persona que requiere cuidados, y de la actitud y tiempo de la persona que cuida. En general, consiste en apoyar la realización de actividades de autocuidado o de gestión. Una característica del cuidado informal o familiar es que se ofrece desde la cercanía, lo cual significa que tiene gran valor para la persona que lo recibe (Imserso, 1995), aunque suponga mayor carga en ocasiones para el/la cuidador/a, como también ha estudiado Inmaculada Zambrano (2006).

Castón y Ramos (2006) resaltan el tipo de cuidados que las personas con dependencia reciben. Los cuidados familiares consisten en la "atención prestada a personas en situación de dependencia en su domicilio, por personas de la familia o de su entorno no vinculadas a un servicio de atención profesionalizada". Los investigadores se refieren a este tipo de ayuda como cuidados informales (Casado y López, 2001).

\section{La dependencia en España y Andalucía}

Los cambios demográficos y sociales en España están generando un incremento progresivo de la población en situación de dependencia. Por una parte, es necesario considerar el importante crecimiento de la población de más de 65 años, que se ha duplicado en los últimos treinta años, pasando de 3,3 millones de personas en 1970 a más de 6,6 millones en 2000, a lo que hay que añadir el fenómeno demográfico denominado 'envejecimiento del envejecimiento', es decir, el aumento del colectivo de población con edad superior a 80 años, que se ha duplicado en sólo 20 años (García Herrero, 2006).

Ambas cuestiones conforman una nueva realidad de la población mayor, que conlleva problemas de dependencia en las últimas etapas de la vida para un colectivo de personas cada vez más amplio. Asimismo, diversos estudios ponen de manifiesto la correlación existente entre la edad y las situaciones de discapacidad, como muestra el hecho de que más del $32 \%$ de las personas mayores de 65 años tengan algún tipo de discapacidad, mientras que ese porcentaje se reduzca a un $5 \%$ para el resto de la población (García Herrero, 2006). Un aspecto de máxima relevancia, tal como señala Fernández Cordón y Tobío (2006), a medio y largo plazo es el importante crecimiento del número de personas mayores de 80 años, susceptibles en mayor medida de sufrir pérdidas y reducciones de su autonomía personal.

Castón (2007) destaca, con respecto a la dependencia, que de los más de tres millones de personas baremadas para su reconocimiento como dependientes en toda España, en Andalucía viven 708.831 ( $10,6 \%$ de la población andaluza), de las cuales el $39,4 \%$ son personas mayores de 65 años. Según los análisis prospectivos del Centro de Estudios Andaluces, los cambios en la dinámica demográfica se mani- 
fiestan ya en un envejecimiento creciente de la población, que se intensificará en el futuro. La proporción de personas de 650 más años seguirá aumentando en el futuro, según todas las proyecciones existentes. En Andalucía, el porcentaje de mayores podría duplicarse de aquí a 2050 (del 14,5\% al 30,9\%) si la inmigración y la fecundidad no se mantienen en los niveles actuales. El grupo de edad que más se incrementará en el futuro es el de los mayores. Las personas de 80 o más años representaban, en 2005 , el 3,4\% de la población, y podrían llegar a ser entre el $7,7 \%$ y el $8,9 \%$ en 2050 , según el escenario considerado. Una carga creciente de dependientes mayores deberá ser soportada por una población en edad de trabaja, que disminuye en términos relativos.

En consecuencia, la mayor longevidad de la población en general ha condicionado el aumento de la prevalencia de las personas con dependencia. Cuando las personas mayores pierden la autonomía en las actividades de la vida diaria, se ponen a prueba los lazos familiares y es cuando se conoce el alcance de la implicación sociofamiliar. Las características estructurales y funcionales del entorno familiar de la persona dependiente juegan un papel tan importante como la propia salud de ésta, sobre todo en la gestión de los procesos de dependencia. Es decir, el número de familiares, los roles que desempeña cada uno y el tipo de cuidado que ofrece cada familia están relacionados con el tipo de prestación que se puede ofrecer a las personas mayores y con la posibilidad de recibir cuidados en su entorno familiar.

\section{Consecuencias de los cuidados prolongados}

El mayor porcentaje de personas cuidadoras en la actualidad son mujeres. Asumir este rol implica una ruptura en la dinámica de la vida cotidiana de la persona cuidadora, en la medida en que el familiar dependiente afectado, sin quererlo, se convierte en el centro que mediatiza todas las actuaciones familiares, tales como el trabajo, el descanso, el ocio o la convivencia familiar. Esta intensa dedicación se agrava según la duración de los cuidados, lo cual puede ocasionar importantes problemas de salud en las personas cuidadoras principales y alterar el equilibrio - personal, de pareja, familiar y social- de quienes rodean a la persona dependiente (Pérez Cano, Musitu Ochoa y Moreno Ruiz 2011).

Uno de los cambios que se observan actualmente en la sociedad tiene que ver con las relaciones interpersonales que se establecen con las redes de solidaridad que se configuran a lo largo de la vida. La evolución económica, demográfica y cultural ha provocado modificaciones, por ejemplo, en la disponibilidad y el tipo de relaciones en distintos momentos de la vida. Diversos estudios alertan sobre la necesidad de planificar desde ahora los servicios sociales para atender las necesidades básicas de las personas dependientes, ya que se prevé un descenso importante de la red de apoyo familiar en las próxi- mas décadas (Imserso y Centro de Investigaciones Sociológicas, 2005). Con la Ley de Dependencia, se intenta dar respuesta a los cambios que se vienen produciendo en la familia, sobre todo a las consecuencias que conlleva la incorporación de la mujer al mercado de trabajo, ya que ésta es la mayor proveedora de servicios y de cuidados de los miembros de la unidad familiar, fenómeno denominado feminización del cuidado (Imserso, 2005).

El propósito general de este artículo es contribuir a la sistematización del conocimiento de las repercusiones de las personas cuidadoras principales de Andalucía que atienden a sus familiares mayores en situación de dependencia, las cuales han sido baremadas y reconocidas con un grado y nivel según la Ley 39/2006, de 14 de diciembre de Promoción de la Autonomía Personal y Atención a las Personas en Situación de Dependencia, desde la perspectiva del análisis del contexto familiar y social en el que se encuentran. Aunque las situaciones de dependencia existen en todos los grupos de edad y también entre aquellas personas que tienen alguna discapacidad, se constata que su incidencia es más considerable entre las personas de mayor edad.

Es en este contexto donde formulamos la pregunta central de la investigación: ¿qué repercusiones tiene la actividad del cuidado de las personas mayores dependientes en las personas cuidadoras principales en los ámbitos físico, psicológico, familiar y social en Andalucía? En concreto, el presente trabajo tiene los siguientes objetivos generales:

- Ampliar el conocimiento de la situación personal (salud física y psicológica), familiar y social de los/as cuidadores/as informales principales que se dedican a atender a personas mayores en situación de dependencia en Andalucía.

- Identificar áreas prioritarias de intervención desde el punto de vista de la salud biopsicosocial en los/as cuidadores/as informales.

La hipótesis de la que partimos la formulamos en los siguientes términos: el cuidado de las personas en situación de dependencia tiene repercusiones negativas en los ámbitos físico, psicológico, familiar y social de las personas cuidadoras principales. Además de esta hipótesis general, se plantean las siguientes hipótesis específicas, relacionadas con la influencia de ciertas variables -estado civil, relación familiar con la persona dependiente, motivos para cuidar, solidaridad o rotación de los familiares- en las repercusiones que tienen en los familiares cuidadores de las personas dependientes:

- El estado civil de las personas cuidadoras principales influye en las consecuencias negativas que tiene la labor del cuidado en los ámbitos físico, psicológico, familiar y social.

- La relación de las personas cuidadoras principales con la persona dependiente (cónyuge, hijo/a, nuera/yerno) influye en las consecuencias negati- 
vas de la labor del cuidado en los ámbitos físico, psicológico, familiar y social.

- Los motivos para ser cuidador/a principal ("porque no me queda otro remedio", "por un deber moral", “por mis creencias"), influye en las consecuencias negativas que tiene la labor del cuidado en los ámbitos físico, psicológico, familiar y social.

- La solidaridad familiar, referida a la rotación de entre varios miembros de la familia para ofrecer los cuidados a la persona dependiente, influye en las consecuencias negativas que tiene la labor del cuidado en los ámbitos físico, psicológico, familiar y social.

\section{Método}

\subsection{Muestra}

La muestra se distribuye proporcionalmente entre las ocho provincias andaluzas, así como entre las zonas urbanas y rurales. Para seleccionar a las personas entrevistadas, se utilizaron dos vías de localización de sujetos: las asociaciones de familiares de enfermos de alzhéimer (dos en cada provincia) y los/as trabajadores/as sociales que realizan proyectos de intervención de dependencia (PIA) ${ }^{1}$ en los domicilios de las personas en situación de dependencia. La muestra está compuesta por 294 familiares que se ocupan actualmente del cuidado de una persona mayor dependiente en la comunidad autónoma de Andalucía. Se partió del supuesto bien conocido de que un alto porcentaje de las personas cuidadoras eran mujeres, de ahí que la distribución por sexo fuera tan absolutamente desigual.

\subsection{Instrumentos}

La metodología utilizada en esta investigación ha sido el análisis de fuentes secundarias y la explotación de la encuesta Entorno Familiar y Dependencia en las Personas Mayores de Andalucía. Con el análisis de fuentes secundarias, se ha pretendido analizar la información existente sobre las repercusiones que la actividad del cuidado de personas mayores tiene en los cuidadores principales. Para ello, se han analizado las siguientes encuestas:

- La Encuesta Nacional sobre Discapacidades, Deficiencias y Estado de Salud (INE, 1999), que permite obtener una visión muy precisa de la situación y las necesidades de las personas que tienen algún tipo de discapacidad, y que, por primera vez, muestra también información sobre

${ }^{1}$ El PIA está regulado en el art. 29 de la Ley de Dependencia. Según se dispone en este precepto, se enmarca en los procedimientos para reconocer las situaciones de dependencia y el derecho a las prestaciones correspondientes. De hecho, el PIA es confeccionado una vez que se dispone de la resolución en la que se reconoce la situación de dependencia. el tipo de ayuda que recibe y las características de ésta, lo que sirve para fundamentar las políticas públicas.

- Encuesta sobre la Soledad en las Personas Mayores (1998), que fue diseñada con el objetivo expreso de caracterizar a la población anciana.

- La Encuesta de Apoyo Informal a los Mayores en España (Imserso, 2004), diseñada para los/as cuidadores/as de apoyo informal y los/as profesionales que trabajan en el cuidado de mayores residentes en hogares.

- Las encuestas sobre uso del tiempo, que contienen información sobre el tiempo dedicado a actividades domésticas dentro del ámbito familiar, entre ellas, las tareas del cuidado de enfermos, mayores y personas con discapacidad.

- La Encuesta de Dependencia y Solidaridad en las Redes Familiares de Andalucía (2005), que se centra particularmente en los cuidadores familiares y sus características. Se estudia el tipo de ayuda proporcionada, así como el tiempo dedicado a ella, aspectos en los que la variable sexo aparece como factor fuertemente explicativo de diferencias.

- La encuesta Condiciones de Vida de las Personas Mayores en Andalucía (2006), que presenta una visión general de la situación que viven los mayores andaluces.

\subsection{Procedimiento}

Para la realización de las entrevistas, se solicitaron las autorizaciones pertinentes a la Consejería para la Igualdad y Bienestar Social, al Ayuntamiento de Sevilla y a las asociaciones de familiares de enfermos de alzhéimer, explicando los objetivos del estudio. Seguidamente se concretó el periodo de administración de los cuestionarios. La cumplimentación de éstos por parte de las personas cuidadoras se llevó a cabo en las asociaciones de enfermos de alzhéimer, en grupos de seis a ocho personas, y en los domicilios de las personas dependientes, bajo la supervisión del encuestador/a o el trabajador/a social.

\section{Resultados}

La metodología cuantitativa consiste en la explotación de la encuesta Entorno Familiar y Dependencia en las Personas Mayores de Andalucía. Las variables de la encuesta fueron sometidas a un análisis factorial de componentes principales con rotación varimax. Finalmente la recogida y tabulación de los datos se realizaron mediante el programa informático estadístico SPSS en su versión 16.0.

Tras el análisis factorial se realizaron análisis univariantes y multivariantes de la varianza (ANOVA y MANOVA, respectivamente) para comprobar si existían diferencias en las repercusiones de la actividad del cuidado, según algunas variables seleccionadas, 
que se engloban en los siguientes grupos: estado civil (solteros/as y casados/as), relación con la persona dependiente (cónyuge, hijo/a, nuera/yerno), motivos para ser cuidador/a ("porque no me queda otro remedio", "por un deber moral”, "por mis creencias"), solidaridad familiar o rotación de las personas cuidadoras (siempre la misma persona cuidadora o por temporadas).

\subsection{Análisis descriptivo de las personas cuidadoras}

Las personas cuidadoras principales son, en su mayoría, mujeres (88,74\%). Dos de cada tres cuidadoras tienen edades comprendidas entre 40 y 60 años. Una de cada cinco tiene más de 60 años. Cuatro de cada cinco son personas casadas, por lo que las consecuencias del cuidado, además de al cuidador o cuidadora, afectan también a la familia con la que conviven. Más de la mitad (55\%) de los/as cuidadores/as tienen estudios primarios o no tienen estudios.

El hecho de que la mayoría de personas encuestadas posean estudios primarios hace que se acentúe el coste del cuidado, debido a que normalmente tienen menos recursos personales para la búsqueda de servicios de apoyo, y por tanto, aumentan las repercusiones negativas que tiene la labor del cuidado en quien lo realiza. El que la hija aparezca también como cuidadora principal tiene implicaciones importantes en su vida como cuidadora, en la medida en que afecta a facetas no sólo familiares, sino también a su vida laboral, tiempo libre y relaciones sociales. Desde que el Imserso elaboró el perfil de la persona cuidadora en 1995, apenas se han destacado cambios en esta figura, cuyo retrato robot sigue siendo el de principios de los años noventa (Imserso, 1995): mujer, en torno a los 55 años, casada, con estudios primarios y sin ocupación remunerada.

\subsection{Análisis factorial}

Según el análisis factorial realizado, los escenarios en los que tiene más implicación el cuidado de las personas dependientes están relacionados con el ámbito físico, psicológico, social y familiar. Los factores que más significado en relación a las variables escogidas para explicar las repercusiones del cuidado están relacionadas con los ámbitos planteados en la hipótesis general (físico, psicológico, familiar y social). El análisis factorial muestra un total de cinco factores. Esta estructura pentafactorial, que recogemos en la Tabla 1, explica el 50,09\% de la varianza.

El factor que más porcentaje de varianza explica es el denominado 'salud psicofísica y social' y es el que más peso tiene dentro de las repercusiones del cuidado, ya que explica el $24,8 \%$ de la varianza. Según la encuesta de Apoyo Informal a las Personas Mayores (2004), el 56\% de las/os cuidadoras/es presentan problemas de salud; un $32 \%$ ha tenido que tomar pastillas; un $25 \%$ no sigue tratamiento, aunque cree que lo necesita; un $20 \%$ ha tenido que ir
Tabla 1. Factores principales de las repercusiones de la labor del cuidado de las personas mayores en los cuidadores principales

\begin{tabular}{|c|c|c|}
\hline Factores & Ítems & $\begin{array}{c}\text { Varianza } \\
\text { explicada (\%) }\end{array}$ \\
\hline \multirow{15}{*}{$\begin{array}{l}\text { Salud } \\
\text { psicofísica y } \\
\text { social }\end{array}$} & Cansancio excesivo & ,785 \\
\hline & $\begin{array}{l}\text { Disminuir el tiempo de } \\
\text { descanso }\end{array}$ &, 767 \\
\hline & Sobrecarga de trabajo & ,758 \\
\hline & Enfermedades (úlceras...) &, 537 \\
\hline & Disminuir el tiempo personal & ,738 \\
\hline & Alteraciones del sueño & ,740 \\
\hline & Disminuir el cuidado personal & ,661 \\
\hline & Lesiones &, 529 \\
\hline & Estrés o ansiedad & ,748 \\
\hline & Disminución de la autoestima & ,612 \\
\hline & Apatía, pérdida de motivación & 699 \\
\hline & Depresión & ,631 \\
\hline & Irritabilidad, cambios de humor &, 517 \\
\hline & $\begin{array}{l}\text { Disminuir relación con los } \\
\text { amigos }\end{array}$ &, 556 \\
\hline & Subtotal & $24,8 \%$ \\
\hline \multirow{8}{*}{ Proyecto vital } & Aumentar las salidas de ocio & 376 \\
\hline & Reducir el tiempo de trabajo &, 781 \\
\hline & Dejar el trabajo & ,811 \\
\hline & Dejar/reducir los estudios &, 721 \\
\hline & $\begin{array}{l}\text { Demorar la propia boda o la de } \\
\text { familiares }\end{array}$ & ,456 \\
\hline & Demorar tener hijos/as &, 648 \\
\hline & Demorar otros proyectos &, 513 \\
\hline & Subtotal & $8,5 \%$ \\
\hline \multirow{6}{*}{$\begin{array}{l}\text { Relaciones } \\
\text { familiares }\end{array}$} & Sentimientos de culpa & ,398 \\
\hline & $\begin{array}{l}\text { Relación conflictiva con persona } \\
\text { enferma }\end{array}$ & ,410 \\
\hline & $\begin{array}{l}\text { Distanciamiento con los/as } \\
\text { hermanos/as }\end{array}$ & ,714 \\
\hline & Ruptura con los/as hermanos/as & ,753 \\
\hline & $\begin{array}{l}\text { Roces frecuentes con los/as } \\
\text { hermanos/as }\end{array}$ & ,639 \\
\hline & Subtotal & $6,7 \%$ \\
\hline \multirow{5}{*}{$\begin{array}{l}\text { Satisfacción } \\
\text { con la tarea }\end{array}$} & Sentirse más fuerte físicamente &, 529 \\
\hline & $\begin{array}{l}\text { Mejorar relación con la persona } \\
\text { enferma }\end{array}$ & ,818 \\
\hline & Más ilusión y ganas de vivir &, 662 \\
\hline & $\begin{array}{l}\text { Satisfacción personal por lo } \\
\text { que hace }\end{array}$ & ,804 \\
\hline & Subtotal & $6,3 \%$ \\
\hline \multirow{6}{*}{$\begin{array}{l}\text { Tiempo y } \\
\text { exigencia para } \\
\text { los/as hijos/as }\end{array}$} & Serenidad y equilibrio personal & ,651 \\
\hline & Más unión con pareja e hijos/as &, 489 \\
\hline & Problemas con pareja e hijos/as &, 385 \\
\hline & Disminuir el tiempo con hijos/as & ,443 \\
\hline & $\begin{array}{l}\text { Más exigencia de pareja e } \\
\text { hijos/as }\end{array}$ & ,390 \\
\hline & Subtotal & $4,6 \%$ \\
\hline Total & & $50,9 \%$ \\
\hline
\end{tabular}

Fuente: Elaboración propia. 
a rehabilitación; un $11 \%$ ha tenido que ir, o va actualmente, a la consulta del psiquiatra o psicólogo; $y$ un $10 \%$ declara que no tiene tiempo de ir al médico (cit. en Zambrano, 2006).

El segundo factor, denominado 'proyecto vital', explica el $8,5 \%$ de la varianza. Se observa que todas las actividades vitales de la persona cuidadora quedan en un segundo plano, se aplazan en el tiempo o no se llegan a realizar, como es el hecho de tener hijos/as, o dejar el trabajo o los estudios. Las primeras renuncias del cuidador o cuidadora están relacionadas con el tiempo de ocio que se suele utilizar con los amigos o vecinos, visitando a otros familiares, por ejemplo.

La persona cuidadora se sobrecarga de las tareas requeridas con la persona dependiente, a costa de disminuir las relaciones sociales, tan necesarias para mantener un nivel aceptable de salud mental. Así, la presencia en actos sociales, como ferias, romerías o bodas, o la realización de actividades tan cotidianas como salir al cine o al teatro, pasear o tomar unas cervezas se va reduciendo cada vez más, de forma que algunos/as cuidadores/as terminan saliendo de casa exclusivamente para cuestiones imprescindibles, como ir al médico, a la farmacia, a hacer las compras, a tirar la basura y muy poco más. Y este estilo de vida, con el tiempo, afecta a la propia personalidad del cuidador o cuidadora.

Hay otros aspectos relacionados con el proyecto de vida, que pueden tener consecuencias más importantes, como dejar los estudios, demorar una boda o la paternidad/maternidad, o dejar el trabajo. Son decisiones que pueden influir de forma casi irreversible en las personas implicadas en el cuidado de un mayor con dependencia. Aun así, no todos las personas cuidadoras manifiestan las mismas reacciones, pues las consecuencias para el/la cuidador/a están mediatizadas por variables de tipo sociocultural.

Estos resultados coinciden con los datos secundarios analizados. Es destacable la falta de tiempo, de vida social, de la personas cuidadoras. Éstas sufren situaciones de este tipo, no pueden plantearse trabajar fuera $(29,7 \%)$, han reducido su jornada $(11,5 \%)$, no cumplen el horario $(11 \%)$, lo que es más radical, han dejado su empleo $(13,2 \%)$ [Rodríguez, 2006].

El tercer factor, llamado 'relaciones familiares' (ítems $16,18,21,22$ y 24 ), explica el $6,7 \%$ de la varianza y alude a la familia de procedencia, formada por los/as hermanos/as, teóricamente con la misma responsabilidad ante el progenitor. La situación del cuidado a veces produce el refuerzo de los lazos familiares, pues se implican, si no todos por igual, al menos cada uno según sus posibilidades. Pero lo más frecuente es que aparezcan conflictos, porque cada uno tiene su punto de vista -al que hay que añadir el del cónyuge-sobre la forma de afrontar el cuidado, y esto hace muy difícil el acuerdo. Suele originar distanciamientos entre hermanos/as y un desgaste en la relación, que, con el tiempo, va pasando una costosa factura en el estado de ánimo de la persona cuidadora o los/as cuidadores/as principales; este estado, sin duda, repercute negativamente en los cuidados que se dispensa a la persona dependiente.

El cuarto factor, denominado 'satisfacción con la tarea', explica el 6,3\% de la varianza y se refiere al sentimiento de obligatoriedad con el que un miembro de la familia (mayoritariamente hijas o esposas) asume un rol en esa familia, y lo hace hasta el término que le produce satisfacción, tal como indican el ítem ('satisfacción personal por lo que se hace') y el ítem ('más ilusión y ganas de vivir').

En la encuesta Dependencia y Solidaridad en las Redes Familiares en Andalucía, se observa que, entre los/as cuidadores/as más jóvenes, la vinculación afectiva con el pariente necesitado de cuidados es una razón más potente que para otros grupos de edad, lo que implicaría que la realización de las tareas de ayuda no sea tanto producto de la interiorización de una responsabilidad o deber para con esos parientes, sino la consecuencia de la buena relación personal. La satisfacción es un elemento que interviene en la emotividad, porque el cariño y el amor también les llevan a luchar por alguien a quien se quiere. En estos casos, el cuidado, a pesar de representar un enorme esfuerzo vital, satisface y hace a las personas cuidadoras sentirse bien consigo mismas.

Al quinto factor se le denominó 'tiempo y exigencia' y explica el 4,6\% de la varianza. En este factor se observa cómo afecta la actividad de cuidado a la familia de los/as cuidadores/as principales que están casados/as y tienen hijos e hijas. En este factor saturan negativamente los ítems referidos a la serenidad y el equilibrio personal, casi imposibles de mantener intactos en los largos procesos de la dependencia actual; y el que hace referencia a la mayor unión con la pareja e hijos. Es decir, no se produce más unión con la pareja e hijos/as, ni tampoco se consigue serenidad. Así, se crean conflictos con la pareja e hijos/ as, debido al tiempo que se les resta, o bien porque muchas veces se lleva a cabo una reorganización de espacios en el domicilio, ya que el familiar afectado con dependencia pasa a convivir con el núcleo familiar.

Cuando la persona mayor se va a vivir a casa de alguno de sus hijos, aparecen muchos conflictos en el hogar de acogida. Esta circunstancia conlleva la redistribución de los recursos familiares, y no sólo los económicos, sino también el propio espacio o el uso del tiempo. La convivencia continuada hace que toda la familia que vive en el hogar se vea afectada por los cuidados que hay que administrar al mayor. En suma, la sobrecarga de los familiares cuidadores viene explicada por las siguientes razones: las amenazas percibidas de debilitamiento o pérdida de su salud física y de su equilibrio personal debidas a la disminución del tiempo para sí mismo; el exceso de responsabilidades, que impide la realización de proyectos vitales propios; el impacto negativo en la familia; la falta de privacidad; y la disminución de las relaciones sociales 


\subsection{Análisis de la varianza}

En relación con el estado civil, se ha observado que el hecho de estar casado o soltero no representa una mayor carga a la hora de cuidar a un mayor dependiente (Tabla 2).

Tabla 2. MANOVA de repercusiones de la actividad de cuidar, según el estado civil de quien la desempeña

\begin{tabular}{|c|c|c|c|c|c|}
\hline \multirow{2}{*}{$\lambda$ de Wilks } & Valor & $\boldsymbol{F}$ & $\begin{array}{c}\text { Grados de } \\
\text { libertad de } \\
\text { la hipótesis }\end{array}$ & $\begin{array}{c}\text { Grados de } \\
\text { libertad } \\
\text { del error }\end{array}$ & Significación \\
\cline { 2 - 6 } & ,975 &, 464 & 5,000 & 90,000 &, 802 \\
\hline
\end{tabular}

Fuente: Elaboración propia.

Respecto del parentesco, es importante subrayar que el $69,90 \%$ de las/os cuidadores/as son hijas/ os; y el $17,30 \%$, cónyuges. El tipo de relación familiar influye en las consecuencias del cuidado, tal y como se puede apreciar en la Tabla 3.
Tabla 3. MANOVA de repercusiones de la actividad de cuidar, según la relación de parentesco entre quien cuida y la persona dependiente

\begin{tabular}{|c|c|c|c|c|c|}
\hline & Valor & $\boldsymbol{F}$ & $\begin{array}{c}\text { Grados de } \\
\text { libertad de } \\
\text { la hipótesis }\end{array}$ & $\begin{array}{c}\text { Grados } \\
\text { de } \\
\text { libertad } \\
\text { del error }\end{array}$ & Significación \\
\cline { 2 - 6 } &, 723 & 3,246 & 10,000 & 184,000 &, 001 \\
\hline
\end{tabular}

Fuente: Elaboración propia.

Para comprobar en qué variables existían diferencias, se realizó el ANOVA correspondiente en todos los factores, observándose diferencias significativas en los factores 'relaciones familiares' $\left(F_{(2,184)}=3,52\right.$, $p<, 05)$ y 'tiempo y exigencia' $\left(F_{(2,184)}=3,83, p<, 05\right)$, como se observa en la Tabla 4.

Finalmente, para conocer entre qué grupos existían diferencias, se realizó la prueba de Tamhane, de diferencias múltiples de medias, que permitió comprobar la relación de la variable dependiente con los factores. Se encontraron diferencias en los factores de 'relaciones familiares' y 'tiempo y exigencia'.

Tabla 4. ANOVA para cada factor del cuestionario de repercusiones de la actividad de cuidar, según la relación de parentesco entre quien cuida y la persona dependiente

\begin{tabular}{l|c|c|c|c|c}
\hline Variable dependiente & $\begin{array}{c}\text { Suma de } \\
\text { cuadrados tipo III }\end{array}$ & Grados de libertad & Media cuadrática & Significación \\
\hline Salud psicofísica y social & 3,674 & 2 & 1,837 &, 129 \\
\hline Proyecto vital &, 954 & 2 &, 477 &, 250 & 3,092 \\
\hline Relaciones familiares & 5,635 & 2 & 2,818 &, 033 \\
\hline Satisfacción con la tarea & 3,965 & 2 & 1,983 & 2,147 \\
\hline Tiempo y exigencia & 6,173 & 2 & 3,086 & 3,830 \\
\hline
\end{tabular}

Fuente: Elaboración propia.

Tabla 5. Medias en los factores del cuestionario de repercusiones de su actividad como persona cuidadora según el parentesco

\begin{tabular}{|c|c|c|c|c|c|c|}
\hline \multicolumn{2}{|c|}{ Parentesco con la persona dependiente } & \multirow{2}{*}{$\begin{array}{c}\begin{array}{c}\text { Salud psicofísica } \\
\text { y social }\end{array} \\
39\end{array}$} & \multirow{2}{*}{$\begin{array}{c}\text { Proyecto vital } \\
20\end{array}$} & \multirow{2}{*}{$\begin{array}{c}\begin{array}{c}\text { Relaciones } \\
\text { familiares }\end{array} \\
23\end{array}$} & \multirow{2}{*}{$\begin{array}{c}\begin{array}{c}\text { Satisfacción con } \\
\text { la tarea }\end{array} \\
46\end{array}$} & \multirow{2}{*}{$\begin{array}{r}\begin{array}{r}\text { Tiempo y } \\
\text { exigencia }\end{array} \\
43\end{array}$} \\
\hline \multirow{3}{*}{ Cónyuge } & Número de casos & & & & & \\
\hline & Media & 3,4304 & 2,2857 & 1,6522 & 2,9239 & 2,2326 \\
\hline & Desviación típica & ,95051 & 1,02598 & ,62149 & ,98583 & ,70634 \\
\hline \multirow{3}{*}{ Hijo/a } & Número de casos & 162 & 106 & 167 & 181 & 152 \\
\hline & Media & 3,5401 & 2,4299 & 2,3353 & 3,1575 & 2,9737 \\
\hline & Desviación típica & ,92308 & 1,37399 & ,92626 &, 86526 & ,88600 \\
\hline \multirow{3}{*}{ Nuera/Yerno } & Número de casos & 10 & 8 & 10 & 12 & 12 \\
\hline & Media & 3,2000 & 2,5893 & 2,2200 & 3,4375 & 3,0167 \\
\hline & Desviación típica & ,80306 & ,71403 & ,89666 & ,61353 & ,96656 \\
\hline \multirow{3}{*}{ Total } & Número de casos & 211 & 134 & 200 & 239 & 207 \\
\hline & Media & 3,5037 & 2,4179 & 2,2510 & 3,1266 & 2,8222 \\
\hline & Desviación típica & ,92238 & 1,29311 & ,91772 & ,88370 & ,90484 \\
\hline
\end{tabular}

Fuente: Elaboración propia. 
Se observa que, respecto a las relaciones familiares, existen diferencias entre el cónyuge (media de 1,65 ) y los/as hijos/as (media de 2,33). La media más alta corresponde a hijos/as, y la más baja, a los cónyuges. El problema estaría en que, entre los/as hermanos/as de la persona cuidadora, se produce distanciamiento, ruptura y roces provocados muy probablemente por las diferencias entre los/as hermanos/as respecto de su compromiso con la persona dependiente y, claro está, en relación a su cuidado.

También se han observado diferencias en función del tiempo y la exigencia, en el sentido de que la relación del parentesco con la persona dependiente altera el equilibrio personal, disminuye la unión con la pareja y los/as hijos/as, y además incrementa el conflicto con la pareja. Estos apartados se reflejan en las medias de los grupos en función del parentesco (cónyuge $=2,23$; hijas $/$ os $=2,97$; y yernos $/$ nueras $=$ $3,01)$ donde la mayor influencia, en este caso negativa, se encuentra en el yerno o la nuera, y la menor, en el cónyuge.

En relación con las motivaciones que subyacen al hecho de ser cuidador, se han realizado análisis descriptivos y MANOVA. Las personas cuidadoras consideran que realizan la labor del cuidado por "un deber moral" (68\%), porque "no les queda otro remedio" $(21 \%)$, o por "coherencia con sus creencias" $(11 \%)$. No obstante, el MANOVA no mostró la existencia de diferencias estadísticamente significativas en los diferentes ámbitos o escenarios de la vida de la persona cuidadora ( $\lambda$ de Wilks $=, 921, p>, 05$ ).

En cuanto a la solidaridad familiar, hemos observado que el $68,88 \%$ de las personas cuidadoras ejercen esta labor sin ayuda de otros miembros de la familia, y que el $31,12 \%$ lo hacen entre varios miembros de la familia, que se turnan por temporadas.

Con respecto a la rotación de las personas cuidadoras tampoco se encontraron diferencias estadísticamente significativas ( $\lambda$ de Wilks $=, 922, p$ > ,05). Este resultado nos sorprende, porque se esperaba que el apoyo familiar tuviera efectos diferenciales en los distintos escenarios considerados en esta investigación y que tienen que ver fundamentalmente con el ámbito físico, psicológico, familiar y social, pero como se observa en los datos secundarios, esta variable también se puede ver influenciada por la edad de la persona cuidadora.

Para seguir profundizando en el análisis sobre si existen diferencias entre las personas cuidadoras principales que realizan esta labor, en solitario 0 compartida con otros miembros de la familia, se procedió a calcular las medias en cada grupo. Los resultados de este análisis tampoco muestran diferencias significativas en ninguno de los ámbitos. No obstante, se observa que las puntuaciones medias más altas corresponden a las personas cuidadoras que ejercen esta labor en solitario, sin ayuda de otros miembros de la familia.
Sintetizando los resultados de las hipótesis específicas de nuestro estudio, podemos destacar que, en relación a los aspectos diferenciales relativos a las dimensiones anteriormente descritas, los efectos del cuidado de las personas mayores en función del estado civil, la relación con la persona dependiente, los motivos para ser cuidador/a y la solidaridad familiar o la rotación del cuidado, solamente la dimensión 'relación con la persona dependiente' es significativa. Se constata que donde más se observa la influencia de esta variable es en los/as hijos/as y, en segundo lugar, en el cónyuge. No haber obtenido diferencias significativas en relación con el estado civil, los motivos para ser cuidador/a y la rotación de las personas cuidadoras induce a seguir profundizando en el estudio y a relacionar otras variables que nos ayuden a explicar las hipótesis planteadas en la Encuesta del Entorno Familiar y Dependencia en las Personas Mayores de Andalucía.

\section{Discusión y conclusiones}

Ante la crisis del apoyo informal que muchos autores describen, se puede concluir que, más que una crisis del apoyo informal, lo que creemos que va a producirse es una transformación de éste. Por un lado, debido a la creciente participación de las mujeres en el mercado de trabajo, lo que probablemente ocurra es que no sólo ellas, sino también sus cónyuges, acaben responsabilizándose de proporcionar los cuidados que el padre o madre de uno de ellos pueda necesitar. Por otro lado, puesto que previsiblemente esas parejas seguirán trabajando a pesar de actuar como cuidadores/as, quizás muchas de ellas traten de buscar un complemento a sus cuidados en la atención domiciliaria. En cualquier caso, para que estos nuevos modos de actuación familiar puedan llegar a producirse, parece evidente que se debe realizarse un requilibrio de fuerzas entre la familia, el sector privado y la Administración.

Revisar la dependencia supone cuestionar una noción cerrada, estrecha, universal y sin visión temporal del análisis de las necesidades de cuidados que tenemos las personas. Además, se propone dar un giro en las connotaciones negativas con que la noción aparece en la esfera pública, en la vida política (Martín-Palomo, 2010). A escala estatal, se observa una tendencia general a solicitar apoyo en el entorno habitual. Ésta es una preferencia lógica y legítima de los usuarios, ya que la institucionalización suele ser el último recurso al que se acude, cuando se han agotado los demás. Aunque permite la permanencia en el entorno habitual, lo hace a costa de afianzar la dependencia familiar, encadenando a cuidadores y personas cuidadas a una relación forzosa que puede desembocar en conflicto (Arnau, 2009; Guzmán, Moscoso y Toboso, 2010).

Sabemos que cuidar a un mayor dependiente en el momento actual es una tarea mucho más difícil y prolongada en el tiempo de lo que era hace dos o tres décadas. Aunque los recursos asistenciales insti- 
tucionales han crecido de forma muy considerable, la dependencia también lo ha hecho. En nuestros días, aproximadamente tres de cada cuatro mayores dependientes siguen estando atendidos por la propia familia. Respecto a la familia del cuidador, su tarea afecta a las relaciones con la pareja y con los hijos, porque la cuidadora, casi siempre mujer, parte del tiempo que dedica al enfermo es a costa del tiempo dedicado a aquéllos. Además, la presencia del enfermo en el ámbito familiar implica no sólo una reorganización de espacios, sino también un cambio, y a veces, una alteración significativa de la convivencia familiar (Musitu y Pérez, 2006).

El estudio realizado nos aporta datos significativos para corroborar la hipótesis general de partida, que el cuidado de las personas en situación de dependencia tiene efectos negativos directos en los ámbitos físico, psicológico, familiar y social de las personas cuidadoras, y además, nos acerca a conocer con mayor detalle el perfil de estas personas, ayudándonos a concretar el grado de afectación al que se ven expuestas.

Como señala Javier Arizkuren (2010), los costes de la dependencia recaen mayoritariamente en la familia, y en concreto, en las mujeres. El nivel de cobertura de servicios sociales, en general, y los de atención a la dependencia, en particular, es bajo si lo comparamos con otros países, especialmente los del norte de Europa. Curiosamente, son estas sociedades las que alaban sistemas como el español, donde el grado de acogimiento y protección familiar es mayor y existe lo que denominamos un 'deber moral en el cuidado de nuestros mayores'. No sólo es una cuestión de ahorro económico, también hay que tener en cuenta que los mayores prefieren, por encima de todo, residir en su casa o con los familiares más próximos, donde se van a encontrar mejor.

Una persona dependiente se convierte en el centro de todas las actuaciones de la familia, el trabajo, el descanso, el tiempo de ocio, de la convivencia familiar. Todo se ve mediatizado por la atención que hay que dispensar al familiar afectado. Las personas cuidadoras principales hacen que toda su vida gire en torno a la atención de las necesidades del familiar dependiente, por ello, terminan padeciendo, indirectamente, pero de forma grave, las consecuencias en las áreas de la vida señaladas en este estudio: salud psicológica y social, proyecto vital, relaciones familiares, satisfacción con la tarea, y tiempo y exigencia.

Los efectos del cuidado de las personas dependientes no difieren en función del estado civil del cuidador o cuidadora. Ésta era la segunda hipótesis del estudio, que no se ha confirmado. En realidad, pensamos que el efecto de cuidar a personas mayores dependientes en los diferentes escenarios de la vida no está relacionado con los recursos que las personas tienen. El hecho de cuidar a un enfermo dependiente es un gran estresor que acaba minando de manera progresiva los recursos de las personas cuidadoras, lo cual se refleja en las diferentes mani- festaciones y estados que estas personas expresan cuando se dedican a esa tarea. Lo que queremos decir es que, probablemente, más que encontrar diferencias en función del estado civil, se debería analizar esto mismo en función de los recursos disponibles, tales como la autoestima, la autoeficacia, la autoconfianza, el apoyo social formal e informal, o la red familiar. Porque, abundando en esta apreciación, una persona dependiente en el hogar es un gran acontecimiento vital negativo $\mathrm{y}$, en consecuencia, un gran estresor, con profundas ramificaciones en los diferentes ámbitos de la vida.

En cuanto a la relación con la persona dependiente, dentro de este escenario de ayuda se observó que las relaciones familiares se ven influidas de manera muy notable, si bien hay diferencias en función del parentesco de la persona que cuida. Cuando se trata del cónyuge, la ruptura, el distanciamiento, los roces con los hermanos y hermanas, la relación -conflictiva o no- con la persona dependiente son menores que cuando la persona que cuida es la hija o hijo, la nuera o yerno. Sorprende en esta situación el hecho de que sea en los/as hijos/as donde más influye la tarea de cuidar, más incluso que en las nueras o yernos. Se puede explicar este resultado desde lo que se podría denominar el sentimiento solidario, que es mucho mayor en la pareja que en los/as hijos/as, y en yernos o nueras.

Es muy probable, como se ha observado en algunos estudios sobre este tema, que el sentimiento de resignación sea mayor en los cónyuges que en el resto de los miembros de la familia. En nuestra experiencia profesional con este tipo de familias hemos podido constatar este hecho y, además, que hijos e hijas se culpabilizan en mucho mayor grado que los cónyuges, debido a que, en sus percepciones, las personas dependientes están bloqueando su desarrollo personal, social y laboral. Desde nuestro punto de vista, el aspecto más destacado de este resultado es que se deben orientar las políticas sociales de apoyo hacia las personas cuidadoras tanto como a las personas dependientes. En otras palabras, es en los hijos e hijas de las personas vulnerables en quienes más directamente influye la tarea del cuidado y, desde la teoría de los recursos, es donde más se agotan éstos y donde más vulnerables son las personas que cuidan.

En consonancia con esta idea, son las nueras, fundamentalmente, las que cuidan a sus suegras o suegros; es en ellas en las que más se perciben dificultades en las relaciones familiares, que se expresan a partir de las demandas y exigencias de los/as hijos/as, en los problemas con la pareja e hijos/as, en la unión con la pareja e hijos/as, y en la serenidad y el equilibrio personal. Es decir, que entre los distintos parentescos entre cuidador/dependiente estos aspectos, tan importantes en la vida de pareja y familiar, son percibidos por las nueras de forma mucho más negativa que por el cónyuge. Parece como si el efecto de la persona dependiente en la persona cuidadora estuviera, al menos parcialmente, influido por el tipo de parentesco. 
Respecto a los motivos para ser cuidador/a, en consonancia con lo que hemos comentado hasta ahora, cabía suponer que diferirían en función de tres grandes categorías, "porque no me queda otro remedio", "por un deber moral" y "por mis creencias", pero lo cierto es que no se han encontrado diferencias significativas entre ellas. Era de esperar que las personas más próximas desde el punto de vista de la relación familiar, como el cónyuge, se diferenciaran de hijos/ as y nueras/yernos en función de sus creencias y del deber moral, y, obvia e inversamente, en relación a la categoría "porque no me queda otro remedio". Nuestras expectativas estaban en que fueran las nueras las que más alto puntuarían y el cónyuge quien más bajo lo hiciera. Es muy probable que no haya diferencias en estas categorías porque se deba a un problema de evaluación.

Probablemente, si se introdujese una escala de valores, como la de Schwartz y Rokeach2, sería muy probable que se encontraran esas diferencias que, en principio, hipotetizábamos. Si esto se confirmase, tendríamos que pensar que no es tanto el parentesco, sino los valores lo que explicaría las motivaciones para el cuidado.

Este aspecto requiere de mayores análisis, y creemos honestamente que sería muy importante desarrollar esta línea de investigación, puesto que, de confirmarse la hipótesis, nos llevaría a orientar, o como mínimo enriquecer, las políticas sociales dirigidas a ayudar a las personas cuidadoras principales. $Y$ esto es importante, porque no es lo mismo estar motivado con una fuerte fundamentación en valores orientados hacia la generosidad, el altruismo o la benevolencia -es decir, valores universalistas-, que hacer esta tarea tan sumamente estresante desde valores mas individualistas como el egoísmo, hedonismo, valores que están en total contradicción con la ayuda a los demás, lo cual, a su vez, es una fuente de estrés y de contradicciones.

En cuanto a la hipótesis de la solidaridad familiar o rotación para los cuidados entre los familiares, no se han encontrado diferencias en ninguno de los

${ }^{2}$ Los valores son "metas u objetivos de carácter general, consistentes a través de las situaciones, que guían la conducta de los seres humanos y están ordenadas por su importancia relativa" (Schwartz, 1992); cuando un conjunto de metas de este tipo goza de una aceptación generalizada en un contexto social determinado, se habla de valores sociales. Así pues, dentro de los valores sociales se incluye un conjunto potencialmente muy amplio de metas relativamente heterogéneas que no tienen todas un carácter normativo equivalente. Rokeach (1973) diferencia entre valores terminales, relativos a estados finales de existencia que se consideran deseables, y valores instrumentales, relativos a modos de conducta positivamente valorados. Dentro de los valores terminales, Rokeach distingue, a su vez, entre valores terminales personales y valores terminales sociales. Los valores terminales personales son aquellos que describen metas que se perciben beneficiosas para el individuo en particular, como 'una vida cómoda' o ‘un sentido de autorrealización'. Los valores terminales sociales son los que representan estados que se perciben deseables para los ámbitos de interacción del sujeto o para la sociedad en su conjunto, como la seguridad familiar la salud y bienestar social y familiar, o 'un mundo en paz'. Dentro de los valores instrumentales, Rokeach diferencia entre valores morales y valores de competencia. (Schwartz, 1992, 1994). escenarios o ámbitos estudiados. Esperábamos que, la rotación de los cuidados entre distintos miembros de la familia influyera en las consecuencias negativas para los cuidadores. Sin embargo la hipótesis no se ha cumplido, lo cual nos ha sorprendido a medias. Por una parte, esperábamos que los miembros de la familia se implicaran más en la ayuda y, en consecuencia, más en los procesos rotatorios, lo cual parece que no es así. Y por otra parte, y de acuerdo con nuestra experiencia profesional, hemos observado que efectivamente la implicación familiar y la solidaridad familiar es mayor cuanto más próximo es el parentesco, aunque, considerando también que es en las primeras fases donde los familiares se solidarizan, se implican y ayudan más, pero que, con el paso del tiempo, los familiares, próximos o lejanos, tienden a desentenderse de esta tarea, abandonando a su soledad a la persona cuidadora principal, que se convierte en única.

Todos estos resultados tienen una gran concordancia con lo que hemos venido comentando hasta ahora, pero este último en particular nos parece de gran trascendencia. El sentimiento de soledad que se genera en las personas cuidadoras, como consecuencia del manifiesto o sutil comportamiento de huida del resto de los miembros familiares, deja a la persona cuidadora en un estado de gran indefensión que tiene implicaciones graves, dependiendo del tiempo de cuidado, de su salud física y psicológica.

Con la técnica utilizada, se ha observado que los factores que se relacionan con las repercusiones de salud psicofísica y social son los que más afectan a las personas cuidadoras principales; menor intensidad o importancia para las personas cuidadoras principales tendrían las repercusiones en sus proyectos vitales, sus relaciones familiares -entre padres e hijos-, sus satisfacciones con respecto a la labor que realizan de cuidados y, finalmente, el tiempo y la exigencia, que afectan a las personas cuidadoras que están casadas en forma de menor atención a sus cónyuges e hijas/os.

Para finalizar, entendemos que este estudio proporciona conocimientos para el desarrollo de otro tipo de investigaciones, no sólo cuantitativas, sino también cualitativas, debido a la importancia de la temática desde la aprobación de la Ley 39/2006, de 14 de diciembre, de Promoción de la Autonomía Personal y Atención a las Personas en Situación de Dependencia.

Como se ha comprobado a lo largo de este estudio, la figura de persona cuidadora no profesional prevista por la ley reconoce una situación muy extendida en nuestro país, dado el predominio de la atención a mayores y dependientes a cargo de las mujeres de sus mismas familias. En noviembre de 2009, según el Imserso habían suscrito el convenio de cuidadores no profesionales 92.897 personas (87.695 mujeres y 5.202 hombres). A este respecto, desde que la ley vio la luz, surgió el interrogante de la posible profesionalización de las cuidadoras frente a la consideración 
tradicional de su papel cuidador. En abril de 2009, el Gobierno anunció su pretensión de que las personas que hubieran carecido de la oportunidad de formarse en el ámbito del cuidado pudieran obtener un título profesional al acreditar experiencia familiar en la atención a menores o familiares dependientes, con el fin de integrarlas en el mercado laboral. (Tobío et al., 2010).

La estimación de posibles beneficiarios que barajaba el Gobierno se situaba en unas 500.000 personas, la gran mayoría mujeres dedicadas al trabajo doméstico, a quienes se acreditaría su competencia profesional como cuidadoras, adquirida a través de la experiencia (Altozano, 2009). Dado el corto espacio de tiempo transcurrido, no hay datos disponibles sobre las consecuencias en el ámbito del cuidado del Real Decreto 1224/2009 de 17 de julio, de Reconocimiento de las Competencias Profesionales Adquiridas por Experiencia Profesional, que fue la medida posterior en la que se tradujo tal objetivo. Por ello, esta línea de investigación está abierta a seguir profundizándose. 


\section{Referencias bibliográficas}

(2006): “Ley 39/2006, de 14 de diciembre, de Promoción de la Autonomía Personal y Atención a las Personas en Situación de Dependencia”, Boletín Oficial del Estado, n-299, 15-12-2006, págs. 44.142-44.156 [<http://www.boe.es/ buscar/doc.php?id=BOE-A-2006-21990>].

(2009): “Real Decreto 1224/2009, de 17 de julio, de reconocimiento de las competencias profesionales adquiridas por experiencia laboral”, Boletín Oficial del Estado, n- 205 , 17-7-2009, págs. [khttp://www.boe.es/buscar/ doc.php?id=BOE-A-2009-13781>].

ALTOZANO, M. (2009): “Titulaciones para las amas de casa”, El País, 26 de abril.

ARIZKUREN, F. J. (2010): “La financiación de la dependencia $\mathrm{y}$ las perspectivas de futuro de las personas mayores en Euskadi”, Zerbitzuan, nㅜㅜ 48, págs. 155-167 [<http://www.zerbitzuan. net/documentos/zerbitzuan/La\%20 financiacion\%20de\%2ola\%2odependencia. pdf>].

CASADO MARÍN, D.; y LÓPEZ I CASASNOVAS, G. (2001): Vejez, dependencia y cuidados de larga duración, colección Estudios Sociales, no 6, Fundación La Caixa [`http://www. imsersomayores.csic.es/documentos/ documentos/casado-vejez-01.pdf`].

CASTÓN, P. (2007): Análisis prospectivo Andalucía 2020. Dependencia, col. Factoría de Ideas, Sevilla, Centro de Estudios Andaluces.

CASTÓN, P.; y RAMOS LORENTE, M. M. (2006): Dependencia en personas mayores en Andalucía, col. Actualidad, Sevilla, Centro de Estudios Andaluces [<http://www. centrodeestudiosandaluces.es/datos/ publicaciones/Actualidado8_final.pdf)].
CENTRO DE INVESTIGACIONES SOBRE LA REALIDAD SOCIAL (1996): Encuesta sobre el Uso del Tiempo, Centro de Investigaciones sobre la Realidad Social.

CENTRO DE INVESTIGACIONES SOCIOLÓGICAS (1998): La soledad en las personas mayores, Madrid, Centro de Investigaciones Sociológicas.

- (1991): Encuesta sobre el Uso del Tiempo, Centro de Investigaciones sobre la Realidad Social.

CENTRO SUPERIOR DE INVESTIGACIONES CIENTÍFICAS (1995): Encuesta sobre Actividades no Remuneradas, Madrid, Centro Superior de Investigaciones Científicas.

EUSTAT (1993): Encuesta de Presupuestos de Tiempo.

FERNÁNDEZ CORDÓN, J. A.; y TOBÍO, C. (dirs.) [2006]: Andalucía. Dependencia y solidaridad en las redes familiares, Sevilla, Instituto de Estadística de Andalucía.

GARCÍA HERRERO, G. (2006): Los servicios sociales y la futura Ley de Promoción de la Autonomía Personal y Atención a las Personas en situación de Dependencia, Federación Andaluza de Municipios y Provincias.

GUZMÁN, F.; MOSCOSO, M.; y TOBOSO, M. (2010): “Por qué la Ley de Dependencia no constituye un instrumento para la promoción de la autonomía personal”, Zerbitzuan, n- 48, págs. 43-56. [«http://www.zerbitzuan.net/documentos/ zerbitzuan/Ley\%2ode\%2odependencia\%20 no\%20constituye\%20un\%2oinstrumento.pdf $>$ ].

IMSERSO (2005), Libro blanco sobre la atención a las personas en situación de dependencia en España, Madrid, Imserso.

- (2004): Encuesta a Empleados y Empleadas de Hogar, Madrid, Imserso. 
- (2004): Encuesta de Apoyo Informal a los Mayores en España, Madrid, Imserso.

IMSERSO; y CENTRO DE INVESTIGACIONES SOCIOLÓGICAS (1995): Las personas mayores en España. Perfiles. Reciprocidad familiar, Madrid, Ministerio de Asuntos Sociales.

IMSERSO; CENTRO DE INVESTIGACIONES SOCIOLÓGICAS; y COLECTIVO IOÉ (1995): Cuidados en la vejez. El apoyo informal, Madrid, Ministerio de Asuntos Sociales.

INSTITUTO DE LA MUJER (2003): Encuesta sobre Mujeres y Trabajo No Remunerado en la Comunidad de Madrid, Madrid, Instituto de la Mujer.

INSTITUTO NACIONAL DE ESTADÍSTICA (2003): Encuesta sobre Discapacidades, Deficiencias y Estado de Salud, Madrid, Instituto Nacional de Estadística.

- (2002-2003): Encuesta de Empleo del Tiempo, Madrid, Instituto Nacional de Estadística.

- (2003): Encuesta sobre Discapacidades, Deficiencias y Estado de Salud, Madrid, Instituto Nacional de Estadística.

- (1999): Encuesta Nacional sobre Discapacidades, Deficiencias y Estado de Salud, Madrid, Instituto Nacional de Estadística.

- (1987): Encuesta sobre Discapacidades, Deficiencias y Minusvalías, Madrid, Instituto Nacional de Estadística.

MARTíN-PALOMO, M. T. (2010): “Autonomía, dependencia y vulnerabilidad en la construcción de la ciudadanía”, Zerbitzuan, nํㅜ 48, págs. 57-69 [<http://www.zerbitzuan.net/documentos/ zerbitzuan/Autonomia,\%2odependencia\%20 y\%2ovulnerabilidad.pdfı].
MONTORO, J. (1999): “Las consecuencias psicosociales del cuidado informal a personas mayores", Revista Internacional de Sociología, no 23, págs. 7-29.

MUSITU, G. et al. (2006): "Apoyo social y calidad de vida en las personas mayores", en PÉREZ CANO, V.; MALAGÓN, J. L.; y AMADOR, L. (dirs.), Vejez, autonomía o dependencia pero con calidad de vida, Madrid, Dykinson.

ORGANIZACIÓN MUNDIAL DE LA SALUD (2002): Envejecimiento activo: un marco político.

PÉREZ CANO, V; MUSITU; y MORENO RUIZ, D. (2011): Familiares cuidadores de mayores: una ardua y silenciosa labor. Realidad y perspectivas de futuro, Madrid, Dykinson.

QUEREJETA, M. (2003): “Discapacidad/Dependencia. Unificación de criterios de valoración y clasificación. Guipúzcoa: Diputación Foral de Guipúzcoa”, Revista Sesenta y Más, ํํ178, págs. 46-51.

RODRÍGUEZ RODRÍGUEZ, P. (2006): El sistema de servicios sociales español y las necesidades derivadas de la atención a la dependencia, Madrid, Fundación Alternativas.

TOBío, C., et al. (2010): El cuidado de las personas. Un reto para el siglo XXI, colección Estudios Sociales, nำ 28, Barcelona, La Caixa [<http://multimedia. lacaixa.es/lacaixa/ondemand/obrasocial/pdf/ estudiossociales/vol28_completo_es.pdf)].

ZAMBRANO, I (2006): La provisión de cuidados a las personas dependientes. El sistema informal de cuidados y el sistema formal para las personas mayores. Informe para el proyecto: Protección Social y Nuevas Necesidades Sociales, Madrid, Ministerio de Educación y Ciencia. 